\title{
A APLICAÇÃO DO BIM NAS SIMULAÇÕES TÉRMICAS DE EDIFICAÇÕ̂ES: UMA REVISÃO SISTEMÁTICA DA LITERATURA
}

\author{
Roberta Oliveira $^{(1)}$, Rodrigo Lameiras ${ }^{(1)}$, Michele Carvalho ${ }^{(1)}$ \\ (1) Universidade de Brasília, Brasília
}

\begin{abstract}
Resumo
O estudo do comportamento térmico e eficiência energética de edificações tem se tornado indispensável nos dias atuais, onde as simulações têm papel fundamental, pois possibilitam a consideração de diversas condições de projeto a fim de encontrar a mais adequada. A implementação do BIM (Building Information Modelling) traz inúmeros benefícios para o processo de projeto como um todo, e pode também ser associada a essa temática. O presente trabalho tem como objetivo realizar uma compilação dos trabalhos na bibliografia técnica que tratam da aplicação do BIM para a realização de simulações térmicas de edificações, cuja lacuna foi detectada por meio de uma busca prévia nas bases de dados Scopus e Web of Science com a geração de uma nuvem de palavras. A metodologia utilizada foi a Revisão Sistemática da Literatura (RSL), utilizando 3 strings de busca, associada a amostragem bola de neve, com a seleção de 18 artigos para análise. Como contribuição, são apresentadas as tendências de pesquisa e as lacunas a serem exploradas, onde nota-se uma tendência categórica quanto à necessidade de redução do tempo e dos esforços demandados no processo de modelagem e simulação, além da utilização do software Autodesk Revit associado a outro programa ou plugin.
\end{abstract}

\section{Introdução}

A eletricidade é essencial para o progresso econômico e a qualidade de vida da humanidade. Ligada à mobilidade social e à saúde, o consumo de energia está crescendo a cada ano no Brasil e no mundo [1]. As edificações em 2018 foram responsáveis pelo consumo de aproximadamente $10 \%$ da energia do país, no qual quase $50 \%$ provém de energia elétrica [2]. Nesse sentido, as estratégias para potencializar o desempenho termoenergético das edificações são um mecanismo para a economia de energia, que podem inclusive proporcionar conforto ao usuário. As decisões de projeto são fundamentais para a escolha de materiais e sistemas construtivos, onde suas propriedades térmicas podem ser analisadas simplificadamente ou por meio de simulações considerando a interação de todos os elementos associados à ventilação natural, orientação das fachadas, entre outros. 
As simulações possibilitam a consideração de diversas condições de projeto durante sua fase inicial a fim de encontrar a mais adequada, além de agilizar esse processo. Entretanto, requerse um nível de detalhamento maior para uma representação mais compatível com a realidade, fato que pode ser proporcionado com a implementação do BIM (Building Information Modelling).

Devido ao interesse dos autores quanto às simulações térmicas de edificações, procurou-se então, aprofundamento no assunto, e por meio de uma busca prévia nas bases de dados Scopus e Web of Science, identificou-se uma lacuna de pesquisas internacionais voltadas para o uso do BIM. Esse resultado foi constatado com a nuvem de palavras gerada pelo programa VOSviewer para a co-ocorrência de termos, a partir da string de busca no título: "thermal AND simulation AND (hous* OR build*)" para artigos em inglês a partir de 2015, resultando em 97 arquivos com a exclusão dos duplicados. A escolha das palavras se deve aos sinônimos mais comumente encontrados nas publicações em língua inglesa para os termos de interesse. A co-ocorrência dos termos está exposta na Figura 1 e foi calculada seguindo as configurações: dados extraídos do título e resumo; contagem binária que fornece o número de documentos em que o termo ocorre pelo menos uma vez; repetição de no mínimo 9 vezes, aproximadamente $10 \%$ dos artigos; $60 \%$ dos termos mais relevantes.

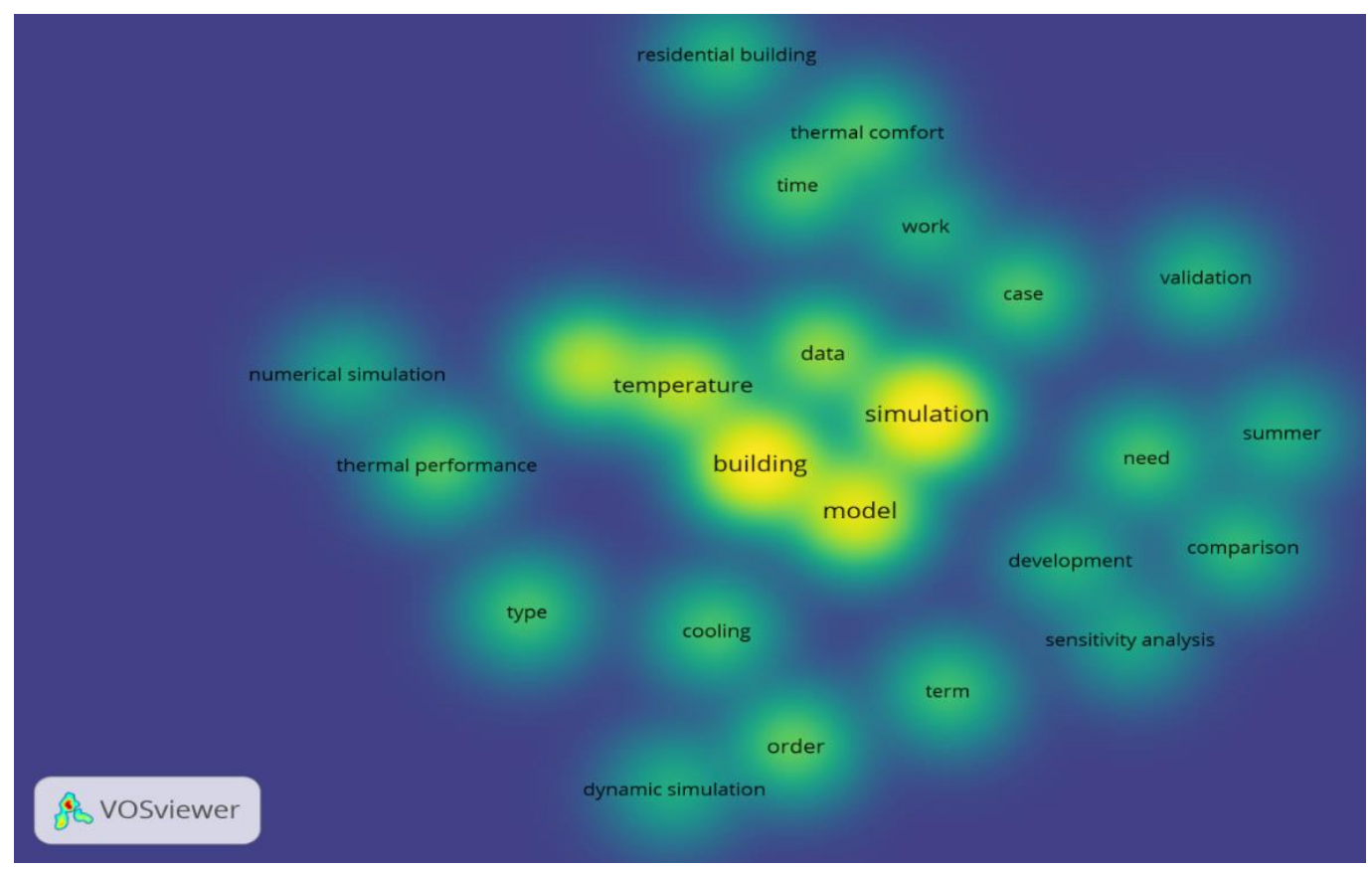

Figura 1: Nuvem de palavras para co-ocorrência dos termos entre os artigos.

Os termos em maior destaque "building" e "simulation" faziam parte da string de busca inicial, além de "temperature", "model" e "data" que são usuais dentro do assunto. Nota-se ainda termos como simulação numérica, dinâmica ou análise de sensibilidade, que possivelmente indicam a metodologia dos artigos. Entretanto, não foi detectada a co-ocorrência do termo BIM ou "Building Information Modelling" conforme se esperava, visto que os artigos são dos últimos 6 anos, período em que os estudos com o BIM tem crescido e demonstrado sua imprescindibilidade no processo de projeto, construção e gestão. 
Embora o BIM esteja bastante difundido, seu potencial não é explorado totalmente quanto às suas capacidades projetuais [3]. Além disso, ainda que exista a oportunidade da simulação térmica de diferentes cenários de forma a encontrar a melhor alternativa, ela ainda não é bem aproveitada [4]. A não utilização também está relacionada à falta de conhecimento dos usuários. O processo BIM fornece aos usuários uma forma de avaliar diversas medidas de melhoria de eficiência energética evitando a remodelagem da geometria e propriedades térmicas da envoltória do edifício, zonas e características dos sistemas de ventilação e climatização necessários para uma análise energética completa [5].

Diante desse cenário, o presente trabalho tem como objetivo realizar uma compilação dos trabalhos na bibliografia técnica que tratam da aplicação do BIM para a realização de simulações térmicas de edificações, através de uma Revisão Sistemática da Literatura (RSL).

\section{Metodologia}

O método utilizado para esse trabalho foi a RSL, cujos desdobramentos permitem uma visão robusta da área de estudo em análise, pois se apresenta como um mecanismo para identificar, avaliar e interpretar toda pesquisa relevante e disponível relativa a um determinado tópico de interesse [6]. Além disso, evita novas pesquisas desnecessárias, inapropriadas, irrelevantes ou antiéticas [7].

Foram utilizadas as mesmas bases de dados da nuvem de palavras: Scopus e Web of Science. Manteve-se o mesmo padrão de busca, acrescido dos termos de interesse: "thermal AND simulation AND (hous* OR build*) AND (Building Information Modelling OR BIM)"; artigos em inglês a partir de 2015, sendo classificada como Etapa 1. Dos 97 artigos pré-selecionados com a string usada na nuvem de palavras, apenas 5 apresentaram os termos "Building Information Modelling" ou "BIM" no título, considerados relevantes no artigo. Entretanto, desses 5, apenas 3 artigos estavam disponíveis.

Optou-se então por expandir as strings: "thermal AND (hous* OR build*) AND (Building Information Modelling OR BIM)" e "thermal AND (Building Information Modelling OR BIM)", com as mesmas restrições (Etapa 2). A primeira retornou 10 artigos, dos quais 9 foram aderentes, e a segunda retornou mais 1 artigo. Os artigos selecionados também trazem em suas discussões a simulação térmica de edificações embora não tenham o termo no título.

Por fim, para eliminar o viés, utilizou-se a amostragem bola de neve, técnica não probabilística de identificação de indivíduos ocultos da população (Etapa 3). Realizou-se então uma busca por artigos de revistas nacionais e também nos anais das edições anteriores do "Congresso Português de Building Information Modelling - ptBIM". O ano de publicação foi mantido, 2015 a 2020, entretanto a busca nos títulos foi mais flexível admitindo-se termos similares aos que foram pesquisados em inglês na tentativa de serem encontrados mais artigos. Incluiu-se ainda um artigo pertencente a um dos autores, que se encaixa no tema de interesse embora não possua os termos no título. Houve a aderência de mais 5 artigos, totalizando 18 a serem explorados.

\section{Discussão dos artigos}

Parte-se então para o resumo e análise dos 18 artigos selecionados na fase anterior, cujos dados iniciais extraídos estão dispostos na Tabela 1. 
Tabela 1: Dados iniciais extraídos dos artigos selecionados

\begin{tabular}{|c|c|c|c|c|}
\hline Seleção & Autores & Idioma & Ano & Meio de publicação \\
\hline \multirow{3}{*}{$\begin{array}{l}\text { RSL: } \\
\text { Etapa } 1\end{array}$} & Kim et al. [8] & Inglês & 2019 & Advances in Civil Engineering \\
\hline & Jeong e Kim [9] & Inglês & 2016 & Sustainability \\
\hline & Kim et al. [10] & Inglês & 2015 & Automation in Construction \\
\hline \multirow{10}{*}{$\begin{array}{l}\text { RSL: } \\
\text { Etapa } 2\end{array}$} & Natephra et al. [11] & Inglês & 2017 & Building and Environment \\
\hline & Ma, Liu e Shang [12] & Inglês & 2018 & Sustainability \\
\hline & $\begin{array}{l}\text { Natehpra, Yabuki e Fukuda } \\
\text { [13] }\end{array}$ & Inglês & 2018 & Building and Environment \\
\hline & Ham e Golparvar-Fard [14] & Inglês & 2015 & Automation in Construction \\
\hline & Garcia e Kamsu-Foguem [15] & Inglês & 2019 & Ecological Informatics \\
\hline & Seigher et al. [16] & Inglês & 2017 & $\begin{array}{l}\text { International Journal of Built } \\
\text { Environment and Sustainability }\end{array}$ \\
\hline & Jeong et al. [17] & Inglês & 2016 & $\begin{array}{c}\text { Journal of Building Performance } \\
\text { Simulation }\end{array}$ \\
\hline & Souza e Tucker [18] & Inglês & 2015 & $\begin{array}{c}\text { Journal of Building Performance } \\
\text { Simulation }\end{array}$ \\
\hline & Liu et al. [19] & Inglês & 2020 & Energies \\
\hline & $\begin{array}{l}\text { Amoruso, Dietrich e Schuetze } \\
{[20]}\end{array}$ & Inglês & 2019 & Sustainability \\
\hline \multirow{5}{*}{$\begin{array}{l}\text { Bola de } \\
\text { neve: } \\
\text { Etapa } 3\end{array}$} & $\begin{array}{l}\text { Queiróz, Grigoletti e Santos } \\
\text { [21] }\end{array}$ & Português & 2019 & $\begin{array}{c}\text { PARC Pesquisa em Arquitetura } \\
\text { e Construção }\end{array}$ \\
\hline & $\begin{array}{l}\text { Silva Junior e Mitidieri Filho } \\
\text { [22] }\end{array}$ & Português & 2018 & $\begin{array}{c}\text { PARC Pesquisa em Arquitetura } \\
\text { e Construção }\end{array}$ \\
\hline & Aljundi, Pinto e Rodrigues [23] & Inglês & 2016 & ptBIM \\
\hline & Miguel e Ferreira [4] & Português & 2016 & ptBIM \\
\hline & Maciel e Carvalho [24] & Inglês & 2019 & Journal of Building Engineering \\
\hline
\end{tabular}

Kim et al. [8] direcionaram o estudo das simulações de desempenho para a otimização da modelagem das janelas e dos cálculos. Propuseram um método para extrair automaticamente, converter e enviar as informações das janelas para um arquivo IFC (Industry Foundation Classes). A modelagem foi feita no programa Autodesk Revit, e as simulações realizadas no THERM. Indicam por fim futuras pesquisas para a utilização de outros programas de simulação. Jeong e Kim [9] e Jeong et al. [17] destacam a adoção da Modelagem Física Orientada a Objetos (OOPM) como uma forma de orientação para a modelagem de objetos capaz de suportar múltiplas simulações de domínio, e da sua utilização nas simulações de desempenho, 
facilitando a interoperabilidade entre BIM e BEM (Modelagem Energética de Edifícios), utilizando a linguagem Modelica. Os modelos foram feitos no Autodesk Revit, mas indicaram novos casos de teste incorporados a outros programas.

A Modelica foi desenvolvida para suportar a modelagem de objetos hierárquicos orientados a objetos de sistemas físicos e generalização de idiomas, além das simulações de vários domínios dentro do mesmo modelo como sistemas mecânicos, termodinâmicos e de controle.

Nesse mesmo sentido, Kim et al. [10] desenvolveram uma biblioteca Modelica BIM com uma abordagem por OOPM para simulações térmicas de edifícios. Para trabalhos futuros, indicam que novas classes de objetos e estruturas de modelos serão investigadas para geometria complexa e sistemas HVAC (aquecimento, ventilação e ar condicionado).

Natephra et al. [11] apresentaram um método para integrar dados térmicos e espaço-temporais no modelo 3D, com a conversão de imagens térmicas coletadas por termografia, integradas ao modelo BIM, calculando assim variáveis de conforto térmico. A aplicação foi feita no Autodesk Revit com o auxílio do programa Rhinoceros e do plug-in Grasshopper. Como estudos posteriores, os autores apontam a aplicação desse método usando os arquivos IFC, e também um sistema de avaliação de conforto térmico dentro do modelo BIM pelo método PMV.

O PMV (voto médio predito), consiste em um valor numérico que traduz a sensibilidade humana ao frio e ao calor. Foi criado por Fanger em 1970, e considera as combinações de variáveis ambientais e humanas para determinação do conforto térmico [26].

Tendo o conforto térmico humano como parâmetro, Ma, Liu e Shang [12] propuseram um sistema baseado em BIM e Rede Neural Artificial (RNA), que comparado ao modelo do PMV, apresentou maior precisão. A integração com o projeto e as informações da construção foi feita por meio de um plug-in desenvolvido em linguagem $\mathrm{C}$ para o Autodesk Revit. Como resultados, tem-se a sensação térmica do indivíduo e o armazenamento dos dados em formato digital para análises futuras, além de fornecer sugestões de otimização de economia de energia.

Natephra, Yabuki e Fukuda [13] apresentam uma abordagem baseada em BIM para fornecer uma avaliação automática dos OTTVs (valor de transferência térmica global) das envoltórias das edificações, extraindo os dados térmicos e físicos do modelo e as propriedades do banco de dados BIM, fornecendo em tempo real a transmitância térmica calculada. Foram utilizados os softwares Autodesk Revit e o Dynamo para a programação visual. É apontado como estudo futuro, a integração do método com os sistemas de certificações ambientais como LEED.

Ham e Golparvar-Fard [14] também propuseram uma associação automática das propriedades térmicas dos elementos modelados em BIM e atualizações baseadas em arquivos gbXML por meio de medições das condições reais de transferência de calor utilizando termografia, a fim de reduzir o tempo e os esforços demandados no processo de modelagem e simulação.

Garcia e Kamsu-Foguem [15] propõe com a ferramenta de mineração de dados e o Autodesk Revit, a previsão dos níveis de conforto baseados em HVAC e do desempenho de um sistema de construção pré-fabricado, visando a melhoria da eficiência energética da edificação.

Seigher et al. [16] desenvolveram uma ferramenta baseada na integração da Linguagem de Programação Visual (VPL) e BIM para o cálculo do valor de transferência térmica da envoltória (ETTV), premissa para a determinação da eficiência energética de algumas certificações. Os softwares utilizados foram o Autodesk Revit, Dynamo e o Microsoft Excel.

Para incentivar e otimizar o cálculo do ETTV, tradicionalmente calculado em planilhas, Liu et al. [19] desenvolveram uma plataforma BIM baseada em nuvem, buscando a certificação verde das edificações. O modelo desenvolvido também permite a atualização automática do índice sob novas atualizações de projeto. 
Souza e Tucker [18] utlizaram o software BPS e estudaram uma lista de possibilidades para os dados de saída das simulações térmicas, sendo possível a escolha de acordo com o objetivo dos projetistas. O objetivo é facilitar a tomada de decisão e melhorar a apresentação ao usuário.

Amoruso, Dietrich e Schuetze [20] elaboraram uma estrutura integrada BIM-paramétrica, para analisar alternativas para o conforto interno antes da reforma de edifícios antigos, com o objetivo de reduzir o tempo de planejamento e custos monetários relacionados. Utilizaram vários programas: Autodesk Revit, Rhinoceros e Grasshopper, EnergyPlus e OpenStudio.

Queiróz, Grigoletti e Santos [21] analisaram o grau de confiabilidade da interoperabilidade para modelos BIM em simulações térmicas. Os modelos digitais foram produzidos no Autodesk Revit e exportados nos formatos de arquivo IFC, gbXML e IDF e convertidos para serem abertos no EnergyPlus. Os autores concluem que não é perfeita essa interoperabilidade, e mantiveram a recomendação do processo de produção de modelos energéticos para o EnergyPlus apartir do plug-in Euclid no SketchUp.

Silva Junior e Mitidieri Filho [22] propõem uma forma de incorporar a verificação dos critérios normativos de desempenho segundo a ABNT NBR 15575 [24] utilizando BIM por meio da verificação de regras e parâmetros (Code Validation) no Autodesk Revit. Para o desempenho térmico, a ferramenta demonstrou potencial de verificação de $100 \%$ dos critérios estabelecidos na norma para o método simplificado (sem simulação). Entretanto, os autores ponderam ainda que os dados podem ser enviados para análise em softwares específicos, como o EnergyPlus.

Aljundi, Pinto e Rodrigues [23] analisaram a confiabilidade e a flexibilidade da análise de energia de uma célula de teste usando simulações baseadas em BIM (com o software Autodesk Revit e o Green Building Studio - GBS), e compararam com os resultados e medições do EnergyPlus para um modelo desenvolvido no SketchUp com o OpenStudio. Como conclusão, destacam que as ferramentas BIM forneceram uma solução integrada com $100 \%$ de interoperabilidade por serem da mesma empresa. Entretanto, o Autodesk Revit apresentou grandes diferenças em relação ao Energy Plus.

Miguel e Ferreira [4] avaliaram a utilização do BIM, com o Autodesk Revit, na certificação energética de edifícios comparada a uma abordagem tradicional, utilizando a ferramenta de análise Hourly Analysis Program, sendo detectados problemas de interoperabilidade e a necessidade de edição manual de parâmetros. Por fim salientam que apesar da disponibilidade de objetos modelados pelos fabricantes, com níveis de desenvolvimento (LOD) elevados, temse a omissão de parâmetros necessários, deixando para o usuário a responsabilidade de definir os mesmos e reunir as evidências necessárias ao seu suporte.

Maciel e Carvalho [24] investigaram o benefício energético de fachadas ventiladas opacas em comparação com fachadas de revestimento no Brasil seguindo a classificação Köeppen-Geiger. Foram utilizadas simulações computacionais para quantificar a energia operacional, com os softwares Autodesk Revit e GBS, sincronizando automaticamente os dados de modelagem e a análise energética de um ano inteiro.

\section{Conclusão}

Por meio de uma busca prévia nas bases de dados Scopus e Web of Science, e pela geração de uma nuvem de palavras com os termos mais relevantes, identificou-se uma lacuna de pesquisas internacionais voltadas para o uso do BIM nas simulações térmicas de edificações, objeto de interesse dos autores. Sendo assim, a metodologia utilizada nesse artigo foi a Revisão 
Sistemática da Literatura nessas bases de dados para artigos dos últimos 6 anos, em inglês, para esse tema. Foram utilizadas três strings diferentes, que retornaram juntas 13 artigos aderentes. Para eliminar ainda mais o viés, utilizou-se a amostragem bola de neve retornando mais 5 artigos, resultando em 18 para a análise.

Todos os artigos selecionados, ainda que com abordagens diferentes, buscaram agilizar e/ou simplificar algum processo para a avaliação do desempenho térmico das edificações e do conforto do usuário. Nota-se uma tendência categórica quanto à necessidade de redução do tempo e dos esforços demandados no processo de modelagem e simulação, proporcionando por exemplo, a atualização automática dos resultados diante de uma alteração de projeto, a fim de acelerar e facilitar a tomada de decisão do projetista.

A utilização do programa Autodesk Revit foi verificada em 13 dos 18 artigos analisados, indicando uma tendência pela facilidade de utilização e edição de parâmetros, ou uma possível restrição com base nos outros softwares BIM do mercado. Dois artigos, no entanto, indicaram como pesquisas futuras o teste das ferramentas desenvolvidas em outros softwares.

A aplicação do Autodesk Revit, na maioria das vezes, esteve associada a programas e plug-ins auxiliares como Rhinoceros, Grasshopper, Dynamo, Green Building Studio Autodesk (GBS) e também EnergyPlus, além da sua associação com Rede Neural Artificial ou Hourly Analysis Program. Foram identificados, contudo, problemas de interoperabilidade entre esses softwares e o Autodesk Revit, com a necessidade de edição manual de parâmetros, distorções detectadas no modelo ou fornecimento de resultados diferentes, sendo minimizados ou inexistentes para aqueles de mesmo desenvolvedor.

Os parâmetros de avaliação para desempenho térmico de edificações no Brasil são estabelecidos na ABNT NBR 15575 [25], que prevê a aplicação do método simplificado, abordado por um artigo, e a simulação utilizando o EnergyPlus, cuja aplicação associada ao Autodesk Revit foi indicada em 4 artigos. Os autores de três deles indicaram diferenças nos resultados obtidos, recomendando a permanência da utilização do EnergyPlus associado a um plug-in (Euclid ou OpenStudio) no SketchUp e a necessidade de averiguação dos métodos de cálculo utilizados por cada um.

Notou-se ainda que alguns autores buscaram associar suas ferramentas além da avaliação de desempenho e conforto termoenergético, mas também à obtenção das certificações ambientais de edificações ou indicaram essa abordagem como pesquisa futura. Tal fato está associado à busca por edificações mais sustentáveis e com consumo baixo ou até nulo de energia, as chamadas zero-energy buildings.

A redução do tempo e otimização das etapas conjuntamente com o aumento da confiabilidade e redução de erros se faz presente em todas as disciplinas de projeto de edificações, e não se demonstrou diferente em se tratando das análises termoenergéticas. A tendência de automatização dos processos para uma análise concomitante ao desenvolvimento do modelo BIM é evidenciada.

Percebe-se assim que, em se tratando de simulação térmica associada ao BIM, não é possível utilizar apenas um software. Logo, a solução dos problemas relacionados à interoperabilidade e à perda de informações é imprescindível, sendo fundamento importante para a aplicação correta do processo BIM.

Do ponto de vista do cenário brasileiro, talvez seja interessante o estudo para aprimoramento do uso do $G B S$ dentro do Autodesk Revit nas simulações, uma vez que, sendo dos mesmos desenvolvedores, a perda de informações seria praticamente nula, na tentativa de obtenção de resultados semelhantes aos emitidos pelo EnergyPlus, respaldado normativamente. Nesse 
sentido, seria eliminada a necessidade de utilização do SketchUp por exemplo, que não possui interface BIM, e sim apenas o desenho 3D.

Constata-se também, pela ausência de artigos, uma lacuna quanto ao nível de detalhamento requerido (LOD) para uma simulação térmica confiável em BIM e de qual o seu impacto nos resultados. Sugere-se realizar esse estudo vinculando também o método de análise de sensibilidade, destacada na nuvem de palavras quando da pesquisa geral dos artigos, mas não detectada em nenhum quando da restrição para os que tratavam sobre BIM.

Os autores procuraram ao máximo expandir os termos de busca para aderência de artigos que discutem o tema. Entretanto, pode-se ampliá-los ainda mais para termos similares na tentativa de haver uma aderência ainda maior de artigos. Ao mesmo tempo, ressalta-se a importância da escolha dos termos que comporão o título dos artigos para que sejam mapeados corretamente em pesquisas que usem a RSL.

Reforça-se assim a relevância desse artigo com a identificação das tendências de pesquisa e das lacunas encontradas para um tema indispensável atualmente (BIM e desempenho termoenergético de edificações) a partir da análise dos artigos, fato que pode evitar pesquisas desnecessárias ou irrelevantes dentro da temática abordada. Além disso, ressalta-se que a RSL pode ser replicada e atualizada pelos leitores a fim de se acompanhar a evolução das pesquisas bem como aprimorá-la.

\section{Referências}

[1] A. C. P. Lopes, D. Oliveira Filho, L. Altoe, J. C. Carlo, and B. B. Lima, "Energy efficiency labeling program for buildings in Brazil compared to the United States' and Portugal's," Renewable and Sustainable Energy Reviews, vol. 66, pp.207-219, 2016. doi: 10.1016/j.rser.2016.07.033.

[2] EPE. Empresa de Pesquisa Energética. Balanço energético nacional. 2019. Disponível em: (http://www.epe.gov.br/pt/publicacoes-dados-abertos/publicacoes/balanco-energeticonacional-2019). Acesso em 13 jan. 2020.

[3] S. Sayegh, "Informações coordenadas", AU - Arquitetura e Urbanismo, vol. 2018, pp.7275, 2011.

[4] R. L. Miguel and D. M. Ferreira, "O BIM na certificação energética de edifícios de serviços", in $1^{o}$ Congresso Português de Building Information Modelling (2016), Universidade do Minho, Guimarães, Portugal, 2016, pp. 601-614. doi: 10.5281/zenodo.166758.

[5] A. Stumpf, H. Kim and E. Jenicek, "Early design energy analysis using building information modeling technology," Relatório Técnico, US Army Corps of Engineers, ERDC/CERL TR-11-41, 2011.

[6] A. Dresch, D. P. Lacerda, and J. A. V. Antunes Júnior, Design Science Research: método de pesquisa para avanço da ciência e tecnologia. Porto Alegre: Bookman, 2015.

[7] D. Gough, S. Oliver, and J. Thomas, An introduction to systematic reviews. Londres: Sage Publications Ltd, 2012.

[8] C. Kim, C. Park, C. Choi, and H. Jang, "Automated Conversion of Building Information Modeling (BIM) Geometry Data for Window Thermal Performance Simulation", Advances in Civil Engineering, vol. 2019, pp. 1-13, 2019. doi: 10.1155/2019/7402089. 
[9] W. S. Jeong and K. H. Kim, "A Performance Evaluation of the BIM-Based Object-Oriented Physical Modeling Technique for Building Thermal Simulations: A Comparative Case Study", Sustainability, vol. 8, pp. 648-674, 2016. doi: 10.3390/su8070648.

[10] J. B. Kim, W. S. Jeong, M. J. Clayton, J. S. Haberl, and W. Yan, "Developing a physical BIM library for building thermal energy simulation", Automation in Construction, vol. 50, pp.16-28, 2015. doi: 10.1016/j.autcon.2014.10.011.

[11] W. Natephra, A. Motamedi, N. Yabuki, and T. Fukuda. "Integrating 4D thermal information with BIM for building envelope thermal performance analysis and thermal comfort evaluation in naturally ventilated", Building and Environment, 124, pp. 194-208, 2017, doi: 10.1016/j.buildenv.2017.08.004.

[12] G. Ma, Y. Liu, and S. Shang. "A Building Information Model (BIM) and Artificial Neural Network (ANN) Based System for Personal Thermal Comfort Evaluation and Energy Efficient Design of Interior Space", Sustainability, 11, 4972, 2019, doi: 10.3390/su11184972.

[13] W. Natephra, N. Yabuki, and T. Fukuda. "Optimizing the evaluation of building envelope design for thermal performance using a BIM-based overall thermal transfer value calculation", Building and Environment, 136, pp. 128-145, 2018, doi: 10.1016/j.buildenv.2018.03.032.

[14] Y. Ham, and M. Golparvar-Fard. "Mapping actual thermal properties to building elements in gbXML-based BIM for reliable building energy performance modeling", Automation in Construction, 49, pp. 214-224, 2015, doi: 10.1016/j.autcon.2014.07.009.

[15] Garcia, L. C., and B. Kamsu-Foguem. "BIM-oriented data mining for thermal performance of prefabricated buildings", Ecological Informatics, 51, pp. 61-72, 2019, doi: 10.1016/j.ecoinf.2019.02.012.

[16] T. E. Seghier, L. Y. Wah, M. H. Ahmad, and W. O. Samuel. "Building Envelope Thermal Performance Assessment Using Visual Programming and BIM, based on ETTV requirement of Green Mark and GreenRE", International Journal of Built Environment and Sustainability, 4(3), pp. 227-235, 2017, doi: 10.11113/ijbes.v4.n3.216.

[17] W. S. Jeong, J. B. Kim, M. J. Clayton, J. S. Haberls, and W. Yan. "A framework to integrate object-oriented physical modelling with building information modelling for building thermal simulation", Journal of Building Performance Simulation, 9(1), pp. 5069, 2016, doi: 10.1080/19401493.2014.993709.

[18] C. B. Souza, and S. Tucker. "Thermal simulation software outputs: a conceptual data model of information presentation for building design decision-making", Journal of Building Performance Simulation, 2015, doi: 10.1080/19401493.2015.1030450.

[19]Z. Liu, Q. Wang, V. J. L. Gan, and L. Peh. "Envelope Thermal Performance Analysis Based on Building Information Model (BIM) Cloud Platform - Proposed Green Mark Collaboration Environment", Energies, 13, 586, 2020, doi: 10.3390/en13030586.

[20]F. M. Amoruso, U. Dietrich, and T. Schuetze, "Indoor Thermal Comfort Improvement through the Integrated BIM-Parametric Workflow-Based Sustainable Renovation of an Exemplary Apartment in Seoul, Korea", Sustainability, 11, 3950, 2019, doi:10.3390/su11143950.

[21] G. R. Queiróz, G. C. Grigoletti, and J. C. P. Santos, "Interoperabilidade entre os programas AutoDesk Revit e EnergyPlus para simulação térmica de edificações", PARC Pesquisa em Arquitetura e Construção, vol. 10, p. e019005, 2019, doi: 10.20396/parc.v10i0.8652852. 
[22] M. A. Silva Junior and C. V. Mitidieri Filho. "Verificação de critérios de desempenho em projetos de arquitetura com a modelagem BIM", PARC Pesquisa em Arquitetura $e$ Construção, vol. 9, n. 4, pp. 334-343, 2018. doi: 10.20396/parc.v9i4.8650453.

[23] K. Aljundi, A. Pinto, and F. Rodrigues, "Energy analysis using cooperation between BIM tools (Revit and Green Building Studio) and Energy Plus", in $1^{\circ}$ Congresso Português de Building Information Modelling (2016), Universidade do Minho, Guimarães, Portugal, 2016, pp. 309-319. doi: 10.5281/zenodo.166758.

[24] A. C. Maciel, and M. T. Carvalho, "Operational energy of opaque ventilated façades in Brazil”, Journal of Building Engineering, 25, 2019. doi: 10.1016/j.jobe.2019.100775.

[25]NBR 15575-1, "Edificações habitacionais: desempenho: parte 1: requisitos gerais", Associação brasileira de normas técnicas (ABNT), 2013.

[26] Labcon. "PMV \& PPD - Conforto higrotérmico", Notas de aula, Universidade Federal do Rio Grande do Sul, 2009, http://www.ufrgs.br/labcon2/aulas_20091/Aula5_PMV_PPD.pdf 